TITLE:

\title{
An easy-to-use single-molecule speckle microscopy enabling nanometer-scale flow and wide- range lifetime measurement of cellular actin filaments.
}

\section{$\operatorname{AUTHOR}(S)$ :}

Yamashiro, Sawako; Mizuno, Hiroaki; Watanabe, Naoki

\section{CITATION:}

Yamashiro, Sawako ...[et al]. An easy-to-use single-molecule speckle microscopy enabling nanometer-scale flow and wide-range lifetime measurement of cellular actin filaments.. Methods in cell biology 2015, 125: 43-59

\section{ISSUE DATE:}

2015

URL:

http://hdl.handle.net/2433/196072

\section{RIGHT:}

(c) 2015 Elsevier Inc. NOTICE: this is the author's version of a work that was accepted for publication in Methods in Cell Biology. Changes resulting from the publishing process, such as peer review, editing, corrections, structural formatting, and other quality control mechanisms may not be reflected in this document. Changes may have been made to this work since it was submitted for publication. A definitive version was subsequently published in Methods in Cell Biology, 125 (2015), doi:10.1016/bs.mcb.2014.10.013; この論文は出版社版でありません。引用の際には出版社版をご確認じ利

用ください。; This is not the published version. Please cite only the published version. 


\section{An easy-to-use single-molecule speckle microscopy enabling nanometer-scale flow and wide-range lifetime measurement of cellular actin filaments}

Sawako Yamashiro, Hiroaki Mizuno, Naoki Watanabe

Department of Pharmacology, Kyoto University Faculty of Medicine, Kyoto, Japan

* Correspondence should be addressed to S.Y. (E-mail: yamashiro.sawako.5c@kyoto-u.ac.jp) Department of Pharmacology, Kyoto University Faculty of Medicine

Yoshida Konoe-cho, Sakyo-ku, Kyoto 606-8501, Japan

Tel: + 81-75-753-4396, Fax: + 81-75-753-4394

Key words: single-molecule speckle microscopy, actin, electroporation, nanometer-scale displacement analysis, computer-assisted tracking of speckles 


\section{Chapter Outline}

\section{Abstract}

1. Introduction

2. Methods

2.1 Preparation of DyLight (DL)-actin probe

2.2 Electroporation for delivery of DL-actin to cytoplasm

2.3 Single-molecule speckle imaging

2.4 Data analysis

3. Perspectives

Acknowledgments

References 


\begin{abstract}

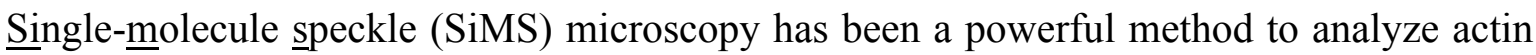
dynamics in live cells by tracking single-molecule of fluorescently labeled actin. Recently we developed a new SiMS method, which is easy-to-use for inexperienced researchers and achieves high spatiotemporal resolution. In this method, actin labeled with fluorescent DyLight dye on lysines is employed as a probe. Electroporation-mediated delivery of DyLight-actin (DL-actin) into cells enables to label cells with $100 \%$ efficiency at the optimal density. DL-actin labels cellular actin filaments including formin-based structures with improved photostability and brightness compared to GFP-actin. These favorable properties of DL-actin extend time window of the SiMS analysis. Furthermore, the new SiMS method enables nanometer-scale displacement analysis with a low localization error of $\pm 8-8.5 \mathrm{~nm}$. With these advantages, our new SiMS microscopy method will help researchers to investigate various actin remodeling processes. In this chapter, we introduce the methods for preparation of DL-actin probes, electroporation to deliver DL-actin, the SiMS imaging and data analysis.
\end{abstract}




\section{Introduction}

Single-molecule imaging is a powerful approach to directly elucidate biochemical reactions in live cells. For example, the approach has provided critical information in remodeling of the actin cytoskeleton (reviewed in Watanabe, 2010), the motion of plasma membrane proteins (Douglass and Vale, 2005), dynamics of microtubule motor proteins (Cai et al., 2009; Ananthanarayanan et al, 2013), transcription factor-DNA interactions in the nucleus (Gebhardt et al., 2013), etc. Although live-cell single-molecule imaging was introduced more than a decade ago, labeling and illumination methods are being improved for further extension of its applications (Gebhardt et al., 2013; Leduc et al., 2013). In this chapter, we introduce a new user-friendly single-molecule imaging of fluorescent actin in live cells.

Actin structures are essential for numerous cellular phenomena, and their dynamics in cells have attracted great attention in the field of cell biology. Live imaging of fluorescently labeled actin

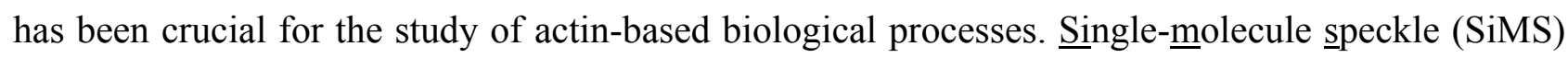
microscopy is a powerful technique for monitoring assembly/disassembly and movement of the actin network (Watanabe, 2012; Watanabe and Mitchison, 2002).

Recently, we developed a new SiMS method, which is easy-to-use for inexperienced researchers and achieves high spatiotemporal resolution (Yamashiro et al., 2014). In this method, we employed an actin probe that is chemically labeled with a fluorescent DyLight dye on lysine side chains. Electroporation-mediated delivery of DyLight-actin (DL-actin) into cells enables to label cells with $100 \%$ efficiency at the optimal low density. DL-actin labels cellular actin networks with improved photostability and brightness compared to GFP-actin. These favorable properties of DL-actin greatly improve the temporal resolution of speckle analysis. The advantage of DL-actin also enables the simultaneous measurement of lifetimes of actin structures with markedly distinct disassembly kinetics. Furthermore, in combination with the Gaussian fit of speckle centroids by Speckle TrackerJ software (Smith et al., 2011), DL-actin enables nanometer-scale displacement analysis with a localization error of $\pm 8-8.5 \mathrm{~nm}$. This allows measurement of the speed of the actin flow within 100-150 nm that is below the diffraction limit of light microscope in live cells.

Before the development of our new SiMS method, several issues had remained unsolved in the study of actin dynamics. First, it was challenging to accurately measure the speed of actin speckles that move only short distances. A previous quantitative Fluorescent Speckle Microscopy (qFSM) study proposing that actin filaments with two distinct retrograde flow rates coexist in lamellipodia (Ponti et al., 2004). This is referred to as the lamella hypothesis because the slower flow speed is equivalent to that in lamella behind the back of lamellipodia. However, another study 
failed to detect such slowly-migrating fluorescent actin speckles in lamellipodia using several speckle tracking approaches (Vallotton and Small, 2009). The authors of the latter study questioned the fidelity of the qFSM method, although both studies (Ponti et al., 2004; Vallotton and Small, 2009) agreed that it remained to be solved whether slowly migrating actin speckles with short lifetimes exist in the lamellipodia. Indeed, filament turnover in the lamellipodial actin network is very rapid, as nearly one third of filaments have short lifetimes of less than $10 \mathrm{~s}$ in certain types of cells (Watanabe and Mitchison, 2002). Those filaments move only short distances $(<100-300 \mathrm{~nm})$, and thereby an exceedingly high spatiotemporal resolution is needed to track short-lived filaments. Now in the retrograde flow velocity measurement with our new nanometer-scale displacement analysis, speckle displacements of 100-150 nm for 3-4 s are sufficient for reliable measurement.

Second, the previous SiMS method demands experience to find cells containing EGFP-actin probes at the optimal level. In the previous method, the low expression level is achieved by the defective cytomegalovirus (CMV) promoter (delCMV), in which the almost entire enhancer region (nucleotides from \#94 to \#547) of the CMV promoter (Enhancer, \#59-465; TATA box \#554-560) was deleted (Watanabe and Mitchison, 2002). However only a minor population of cells expresses EGFP-actin at a sufficiently low level. In contrast, our electropolation method enables to deliver DL-actin to the cytoplasm at a similar low density suitable for SiMS microscopy with almost $100 \%$ efficiency.

Third, there had been a warning about the usage of EGFP-actin for monitoring actin dynamics mediated by formin-homology proteins (formins). Formins are a major family of actin nucleators in the cell, and play critical roles in the contractile ring formation (Severson et al., 2002; Watanabe et al., 2008), assembly of the actin cortex (Bovellan et al., 2014), migration of neurons (Shinohara et al., 2012) and thin filament assembly in myofibrils (Kan-o et al., 2012). Several observations have indicated evidences that suggest a potential problem about usage of EGFP-actin. Fluorescent protein tagged actin, such as EGFP-actin, may not be efficiently incorporated into filaments assembled by formins (Chen et al., 2012; Yamashiro et al., 2014). We prepare DL-actin with special care, and confirmed that DL-actin retains the ability to incorporate into mammalian formin mDial and mDia2-assembled filaments in vitro (Yamashiro et al., 2014). The SiMS method with DL-actin would be useful for investigating the actin dynamics in formin-based actin structures.

Our new SiMS method provides solutions for above problems. The method is user-friendly, and enables to unveil actin kinetics and movements in various actin structures, including formin-based networks. In this review, we describe the methods for preparation of DL-actin probes, delivery of DL-actin to cytoplasm by electroporation, the SiMS imaging and data analysis. We also 
discuss perspectives on the application of our new SiMS microscopy for the cell biological research.

\section{Methods}

\subsection{Preparation of actin probes}

In this section, we describe the procedure for preparing fluorescent actin probes that are chemically labeled with amine-reactive DyLight dyes (Thermo Fisher Scientific Inc.). Especially DyLight 549- and 550-labeled actin probes show greatly improved photostability and brightness in cells. Note that DyLight 550 is the replacement of DyLight 549 that has been discontinued. Fluorescent actin labeled on lysine incorporates into actin filaments elongated by some of formin family proteins in vitro (Kovar and Pollard 2004; Kovar et al., 2006; Michelot et al., 2007; Yamashiro et al., 2014).

For the labeling reaction, we follow the method of Kellogg et al. (Kellogg et al., 1988) with modification. In the original method, F-actin (filamentous actin) is used for the labeling reaction to prevent DyLight dyes from labeling actin at the positions which interfere with polymerization. We strongly recommend further purifying actin by gel filtration after the labeling procedure to remove actin molecules with multiple DyLight dyes. Our mass spectrometry showed that 1 to 3 lysines of actin are labeled with the DyLight dye (Yamashiro et al., 2014). The positions of labeled lysines have been unidentified. In gel filtration chromatography of DL-actin using the Superdex 200 pg HiLoad 16/60 (16×600 mm; GE Healthcare), double- or triple-labeled actin is eluted faster than unlabeled and single-labeled actin (Yamashiro et al., 2014). It is unlikely that DyLight-labeled actin are separated due to the size difference arising from the DyLight dyes ( $\sim 1000$ Da). We speculate that double and triple DyLight-labeled actin tend to form aggregates, and thereby those species might be eluted in the early fractions. Previous studies have shown that actin dimer cross-linked by chemical linker do not harbor the normal properties of actin (Bubb and Korn, 1995; Millonig et al., 1988). In particular, the cross-linked dimer identified in the initial phase of actin polymerization, which is termed the "lower dimer", does not promote actin nucleation (Millonig et al., 1988). Therefore, removing actin aggregates is likely a critical procedure in DL-actin preparation.

DL-actin incorporates into mDial- and mDia2-associated filaments with $77 \%$ and $85 \%$ efficiency of formin-free filaments in the presence of profilin in vitro (Yamashiro et al., 2014). However, it poorly incorporates into FMNL2-assembled filaments with $25 \%$ efficiency of formin-free filaments (Yamashiro et al., 2014). There are fifteen formins in human (Schonichen and 
Geyer, 2010), and the filament incorporation rate of DL-actin may vary with different formins.

\section{Required materials}

- G-actin (globular actin): Prepare from the rabbit skeletal muscle according to the method for Spudich and Watt (Spudich and Watt, 1971). Rabbit skeletal muscle acetone powder (Pel-Freez) is also commercially available, and can be used as a source of G-actin.

- G-buffer: $2 \mathrm{mM}$ Tris-HCl, $0.2 \mathrm{mM} \mathrm{CaCl}_{2}, 0.1 \mathrm{mM}$ adenosine triphosphate (ATP), $1 \mathrm{mM}$ dithiothreitol (DTT), pH8.0

- DTT(-)-Hepes-G-buffer: 5 mM Hepes-KOH, $0.2 \mathrm{mM} \mathrm{CaCl}_{2}, 0.1$ mM ATP, pH7.5

- $10 \times$ Polymerizing solution: $500 \mathrm{mM} \mathrm{KCl}, 20 \mathrm{mM} \mathrm{MgCl}_{2}, 2$ mM EGTA

- 6×Labeling buffer: 300 mM Pipes, 300 mM KCl, 1.2 mM CaCl $2,1.2$ mM ATP, pH6.8

- DyLight NHS Ester $1 \mathrm{mg}$ (Thermo Fisher Scientific Inc.)

- $N, N$-Dimethylformamide (DMF), anhydrous (Sigma-Aldrich)

- Protein Assay CBB solution (Nacalai Tesque, \#29449-15)

\section{Procedure for DyLight NHS Ester labeling of actin}

1. To remove Tris and DTT from the G-actin solution, mix $6 \mathrm{ml}$ of $30 \mu \mathrm{M} \mathrm{G}$-actin $(1.26 \mathrm{mg} / \mathrm{ml}$, total $7.56 \mathrm{mg}$ ) with a $1 / 9$ volume of $10 \times$ polymerizing solution and incubate for $2 \mathrm{hrs}$ at $25^{\circ} \mathrm{C}$.

2. Pellet F-actin (filamentous actin) by centrifugation at $100,000 \times g$ for $1 \mathrm{~h}$ at $4{ }^{\circ} \mathrm{C}$.

3. Rinse the pellet of F-actin gently twice using with cold DTT(-)-Hepes-G-buffer. Then, dissolve the pellet in $6 \mathrm{ml}$ of DTT(-)-Hepes-G-buffer and dialyze against $500 \mathrm{ml}$ of DTT(-)-Hepes-G-buffer overnight at $4{ }^{\circ} \mathrm{C}$. During dialysis, change the buffer at least once.

4. After dialysis, measure the concentration of G-actin by using the Protein Assay CBB solution or by absorption at $290 \mathrm{~nm}$ according to the equation:

G-actin $(\mathrm{mg} / \mathrm{ml})=(\mathrm{A} 290-\mathrm{A} 320) / 0.63($ Houk and Ue, 1974)

5. Polymerize dialyzed G-actin $(\sim 30 \mu \mathrm{M})$ by adding $1 / 5$ volume of $6 \times$ labeling buffer for 2 hrs at 25 ${ }^{\circ} \mathrm{C}$ in a conical centrifuge tube. Wrap the tube with aluminum foil.

6. Before use, dissolve $1 \mathrm{mg}$ DyLight NHS Ester (DyLight 550 NHS Ester, M.W. 1040.06) with 24 $\mu \mathrm{DMF}$ to make $40 \mathrm{mM}$ stock solution.

7. Add the DyLight stock solution to F-actin in a conical tube (prepared at the step 5) at a final concentration of $120 \mu \mathrm{M}$. Mix immediately by inverting the tube several times. Incubate on a rotating wheel for $2 \mathrm{hrs}$ at room temperature or overnight at $4{ }^{\circ} \mathrm{C}$. Use a rotating wheel at slow speed to prevent the actin solution from bubbling. 
8. Stop the reaction by adding Tris- $\mathrm{HCl}(\mathrm{pH} \mathrm{8.0)}$ at a final concentration of $10 \mathrm{mM}$, and then remove aggregates in the mixed solution by centrifugation at $3,200 \times g$ for 10 min at $4{ }^{\circ} \mathrm{C}$. Collect the supernatant.

9. Pellet the labeled F-actin by centrifugation at $100,000 \times g$ for $1 \mathrm{~h}$ at $4{ }^{\circ} \mathrm{C}$.

10. Dissolve the pellet in $2 \mathrm{ml}$ of cold G-buffer and dialyze against $500 \mathrm{ml}$ of G-buffer in a beaker wrapped with aluminum foil overnight at $4{ }^{\circ} \mathrm{C}$. During dialysis, change G-buffer at least once.

11. Remove unsolved aggregates in labeled G-actin solution by centrifugation at $100,000 \times g$ for $1 \mathrm{~h}$ at $4{ }^{\circ} \mathrm{C}$.

12. Mix the supernatant with a $1 / 9$ volume of $10 \times$ polymerizing solution and incubate for $2 \mathrm{hrs}$ at 25 ${ }^{\circ} \mathrm{C}$.

13. Repeat the steps 9-11 once again. Those steps are important to remove denatured actin molecules and extra dyes.

14. Purify labeled G-actin by gel filtration using the Superdex 200 pg HiLoad 16/60 (16×600 mm; GE Healthcare). Run G-buffer through column and collect $2 \mathrm{ml}$ fractions in the dark at $4{ }^{\circ} \mathrm{C}$. To perform the gel filtration chromatography, we use an AKTAprime plus liquid chromatography system (GE Healthcare). Use DL-actin in the back part of the total G-actin peak in further experiments.

15. Determine the concentration of total G-actin in the purified DL-actin solution by measuring the absorbance at $290 \mathrm{~nm}$. Also, determine the concentration of dye bound to G-actin by measuring the absorbance at the wavelength of maximum absorbance of the DyLight dye. Calculate the degree of labeling by dividing the concentration of DyLight dye by the concentration of total G-actin. G-actin thus prepared is usually $\sim 10 \%$ labeled.

16. DL-actin in G-buffer is stored on ice in the dark for a few weeks. If long-term storage is required, DL- actin is divided to small aliquots, quickly frozen in liquid nitrogen and stored at $-80{ }^{\circ} \mathrm{C}$.

\subsection{Electroporation method for delivery of DL-actin to XTC cells}

The electroporation method achieves incorporation of DL-actin into almost $100 \%$ of Xenopus XTC cells at the sufficiently low-density for SiMS imaging (Yamashiro et al., 2014). We have successfully carried out electroporation of DL-actin with mammalian MDCK cells and Xenopus epithelial A6 cells in the same method with XTC cells. We use the Neon ${ }^{\mathrm{TM}}$ transfection system (Invitrogen). The maintenance of XTC cells is described in our previous article (Watanabe, 2012). Before electroporation, monomeric DL-actin is mixed with profilin that prevents 
spontaneous filament nucleation during the procedure. We do not observe any apparent cell damage after electroporation, as the morphology and actin organization are indistinguishable between DL-actin-loaded cells and control cells without electroporation. DL-actin labels bright vesicle-like structures (Fig. 1A, an arrow in the middle image). We speculate that the structure is an artifact arising from endocytosis of DL-actin during electroporation. Performing electroporation at low temperatures $\left(4-10^{\circ} \mathrm{C}\right)$, which suppresses endocytosis, reduces appearance of the bright vesicle-like structures in DL-actin-loaded cells. We recommend performing electroporation with a pre-cooled buffer (Electrolytic Buffer E, Neon ${ }^{\mathrm{TM}} \mathrm{Kit}$ ) and electroporation samples, and then washing the cells with pre-cooled medium. The DL-actin-loaded cells retain actin speckles for SiMS microscopy up to 3 to 4 days.

\section{Required materials}

- DyLight-labeled G-actin (see Section 2.1)

- profilin: we use recombinant human profilin I purified as previously described (Mizuno et al., 2011).

- The Neon ${ }^{\mathrm{TM}}$ transfection system $10 \mu \mathrm{l}$ kit (Invitrogen)

- 70\% Leibovitz's L-15 medium containing 10\% fetal bovine serum (FBS): add $150 \mathrm{ml}$ sterile water (v/v) and $50 \mathrm{ml} \mathrm{FBS} \mathrm{to} 350 \mathrm{ml}$ of L-15 medium (Gibco, \#11415-064).

- $70 \%$ Trypsine-EDTA: add sterile water $30 \%(\mathrm{v} / \mathrm{v})$ to the $0.25 \%$ trypsin $1 \mathrm{mM}$ EDTA solution (Nacalai tesque, \#35554-64)

\section{Procedure for electroporation of DL-actin into XTC cells}

1. Mix $15 \mu \mathrm{M}$ profilin and $5 \mu \mathrm{M}$ G-actin (5-15\% DL-labeled) in $40 \mu \mathrm{l}$ G-buffer by repeated pipetting. Incubate the mixture on ice for $30 \mathrm{~min}$.

Note: The concentration of DL-actin is optimized for observation of the actin network in lamellipodia of XTC cells. Adjust its concentration depending on cell types and the actin structures of interest.

2. To remove aggregates, centrifuge the mixture at $300,000 \times g$ for $1 \mathrm{~h}$ at $4{ }^{\circ} \mathrm{C}$. Collect upper half of the supernatant and keep the mixture on ice in the dark.

3. Trypsinize and then resuspend XTC cells in 70\% L-15 medium containing FBS to inactive trypsine. Collect cell pellets by centrifugation at $1000 \mathrm{rpm}$ for $1 \mathrm{~min}$. Resuspend cells in $1 \mathrm{ml}$ of 70\% L-15 medium without FBS. 
4. Count the number of cells. Typically, $\sim 2 \times 10^{6}$ cells are harvested from one T-flask $\left(25 \mathrm{~cm}^{2}\right)$ with confluent XTC cells.

5. Collect $9.3 \times 10^{5}$ cells and centrifuge at $1000 \mathrm{rpm}$ for $1 \mathrm{~min}$ to pellet cells. Remove medium.

6. Prepare the electroporation solution $(50 \mu \mathrm{l})$ by mixing $45 \mu \mathrm{l}$ Resuspension Buffer R (Neon $\left.{ }^{\mathrm{TM}} \mathrm{Kit}\right)$ and $5 \mu \mathrm{l}$ of the DL-actin-profilin mixture. Resuspend the cells with $50 \mu \mathrm{l}$ of the electroporation solution. Mix well by repeated pipetting and keep the cell suspension at $4{ }^{\circ} \mathrm{C}$.

7. Transfer $10 \mu \mathrm{l}$ of the cell suspension into an electroporation pipet tip (10 $\mu 1)$, and then apply two pulses $(1005 \mathrm{~V}, 35 \mathrm{~ms})$.

8. Transfer the cells in the tip to $5 \mathrm{ml}$ of $70 \% \mathrm{~L}-15$ medium without FBS pre-cooled at $4{ }^{\circ} \mathrm{C}$ in a 15 ml conical tube. Keep the tube at $4{ }^{\circ} \mathrm{C}$.

9. Repeat Steps 7 and 8 four times. You can use the same electroporation pipet tip repeatedly.

10. To wash the cells, collect cell pellets by centrifugation at $1000 \mathrm{rpm}$ for $1 \mathrm{~min}$, and then resuspend with $5 \mathrm{ml}$ of $70 \% \mathrm{~L}-15$ medium without FBS at $4{ }^{\circ} \mathrm{C}$. Repeat this step at least three times.

Note: Wash the cells thoroughly. Otherwise the remained extra DL-actin in the solution may interfere with single-molecule imaging by sticking on the glass coverslips.

11. After the last washing step, resuspend the cells with 2-3 ml of 70\% L-15 medium without FBS at $23{ }^{\circ} \mathrm{C}$. XTC cells efficiently spread on coverslips in the medium without FBS. The cells are ready for observation as Section 2.3. The rest of the cells can be maintained in a flask with $70 \%$ L-15 medium containing FBS for 3-4 days.

Note: The Step 11 describes the procedure optimized for XTC cells. Change the conditions depending on cell types.

\subsection{Single-molecule speckle imaging}

In this section, we briefly illustrate the method of the SiMS imaging with DL-actin loaded cells. See also our previous article (Watanabe, 2012) in that we described the protocol for SiMS observation in detail.

DL-actin speckles incorporate into cellular actin structures including lamellipodia, stress fibers and filopodia (Fig. 1A). DL-actin also labels bright vesicle-like structures, which might be an artifact arising from endocytosis of DL-actin during electroporation (Fig. 1A, arrow). Because such vesicle-like structures are markedly larger and brighter than single-molecule speckles and they move stochastically changing directions, they can easily be distinguished from DL-actin speckles in the cytoplasm and eliminated from the analysis. 
Glass coverslips are coated with poly-L-lysine (PLL) alone or doubly with PLL and extracellular matrix (ECM) proteins. It is important to choose proper coating, because XTC cells form distinct actin organization depending on molecules attached to coverslips (Yamashiro and Watanabe, in press, 2014). To coat a coverslip, the coverslip is incubated with the distilled water containing each coating molecule, and then the excess amount of the molecule is removed and extensively washed away with the distilled water. When the coverslip is prepared with $1 \mathrm{mg} / \mathrm{ml}$ PLL, XTC cells rapidly form wide and flat lamellipodia, which allows long-range single-molecule tracking in a single focal plane. When the coverslip is prepared with $10 \mu \mathrm{g} / \mathrm{ml}$ PLL and $10 \mu \mathrm{g} / \mathrm{ml}$ fibronectin sequentially, XTC cells form narrow lamellipodia at the cell periphery, focal adhesions at the base of lamellipodia, and well-developed stress fibers. On the other hand, wide lamellipodia containing elongated focal adhesions are formed in XTC cells culture on coverslips prepared with $0.1 \mathrm{mg} / \mathrm{ml}$ PLL and $5 \mu \mathrm{g} / \mathrm{ml}$ laminin sequentially.

We recommend starting with SiMS time-lapse imaging for the lamellipodial actin network moving with the retrograde flow (Fig. 1B), which is easy to track. Adjust the level of the excitation light if necessary. Strong illumination rapidly decreases the speed of actin retrograde flow due to photo damage to the cell (Millius et al., 2012; Yamashiro et al., 2014). Keep in mind to minimize photodamage by minimizing irradiation of excitation light during focus adjustment and image acquisition. This can be achieved by carefully selecting neutral density filters (Watanabe, 2012) and restricting the area by diaphragm.

\section{Required materials}

- DL-actin loaded XTC cells (see Section 2.2)

- Glass coverslips (Matsunami No.1, thickness $0.12-0.17 \mathrm{~mm}$, circular $24 \mathrm{~mm}$ diameter)

- $1 \mathrm{mg} / \mathrm{ml}$ Poly-L-lysine (PLL) (Sigma; P2636)

- Cell chambers: handmade flow cells (Watanabe, 2012) for the upright microscope or Attofluor cell chambers (Life technology; A7816) for the inverted microscope

\section{Examples of microscopy setups}

SiMS imaging can be achieved using epi-fluorescence microscopy equipped with a high sensitivity charge-coupled device (CCD) detector and high magnification objective lenses with a large numerical aperture. Under low light conditions, EM-CCD cameras work better than other detectors, but single-molecule imaging can also be achieved using cooled CCD cameras. Total 
internal reflection fluorescence (TIRF) system is effective for imaging SiMS near the plasma membrane with low background fluorescence (Millius et al., 2012). See also our previous article (Watanabe, 2012) in that we described microscopy setups for SiMS imaging in detail. [A]-[C] are the microscopic setups used for SiMS imaging in our laboratory.

[A] Olympus IX71 microscope equipped with a Photometrics Evolve 512 CCD camera, UPlanSApo NA1.4 100× objective lens, and UPLSAPO NA1.3 60× silicone oil objective lens.

[B] Olympus BX52 microscope equipped with a Roper Cascade II 512 CCD camera and UPlanSApo NA1.4 100× objective lens.

[C] Olympus IX83 microscope equipped with a Photometrics Evolve 512 CCD camera, a TIRF illumination system (Olympus, IX2-RFAEVA-2), custom optical shutters with acousto-optics, tunable filters, Cobolt Blues ${ }^{\mathrm{TM}} 473 \mathrm{~nm}$ DPSS laser, a $488 \mathrm{~nm}$ laser (Newport, PC14584) and a UApoN NA1.45 100× TIRF objective lens.

\section{Procedure for the SiMS imaging of DL-actin loaded XTC cells}

- Preparation of PLL-coated coverslips

1. Soak glass coverslips with detergent for lab glassware (SCAT 20X-PF etc.) in distilled water $(1: 19)$ and shake gently for more than $1 \mathrm{~h}$ at room temperature.

2. Wash coverslips extensively with distilled water.

3. Dispense droplets $(\sim 60 \mu \mathrm{l})$ of the distilled water containing $1 \mathrm{mg} / \mathrm{ml}$ PLL on a sheet of parafilm. Overlay the washed coverslip over the droplet. Cover the coverslips with another sheet of parafilm. Seal the edge of parafilm by pressing down the two sheets with nails to avoid evaporation of the PLL solution. Keep at room temperature at least for $1 \mathrm{~h}$. The PLL-coated coverslips can be stored at room temperature, and are usable for several days unless they are dried.

4. Before use, soak the coverslips with distilled water with the PLL-coated face up. Wash coverslips extensively with distilled water. To coat the PLL-coated coverslips further with ECM proteins, repeat the steps 3 and 4 with the ECM solution instead of the PLL solution.

\section{- Observation}

1. Assemble a handmade flow cell or an Attofluor cell chamber with a PLL-coated coverslip. The handmade flow cell is especially adequate for rapid perfusion of the medium to observe drug effects on molecular behavior (Watanabe, 2012). The Attofluor cell chamber is suitable for long-term observation. 
2. Transfer $500 \mu \mathrm{l}$ of the DL-actin loaded XTC cell suspension (see Section 2.2, step 11) into the assembled flow cell or the cell chamber.

3. Incubate for $30 \mathrm{~min}$ at room temperature in a dark, humidified container.

4. Set the chamber on the microscope with high magnification objective lens $(60 \times, 100 \times, 150 \times)$ and set focus to cells.

5. Acquire single DL-actin molecule imaging using epifluorescent illumination system. To visualize DL550-actin, time-lapse imaging is performed using the microscope setup [A] above with the $100 \mathrm{~W}$ mercury excitation attenuated to $1.5 \%$ using neutral density filters and with a $2 \mathrm{~s}$ exposure time (Fig. 1B and Fig. 2). Adjust the level of the excitation light and the exposure time as necessary.

\subsection{Data analysis}

In this section, we describe nanometer-scale displacement analysis in cells and the simultaneous lifetime measurement of actin populations with markedly distinct disassembly kinetics. The imaging analysis tool, Speckle TrackerJ (Smith et al., 2011), has been developed by our collaborator Dimitrios Vavylonis and his colleagues at Lehigh University. Speckle TrackerJ combines manual editing and computer assisted techniques to accurately detect and track speckles. The tool is a freely available open source ImageJ plugin (http://athena.physics.lehigh.edu/speckletrackerj/index.chy). The method to use Speckle TrackerJ is described on the above website.

(i) Nanometer-scale displacement measurement

In combination with Speckle TrackerJ, DL-actin enables measurement of speckles' centroids with a low localization error of $\pm 8-8.5 \mathrm{~nm}$ at $100 \mathrm{~ms}$ intervals. Two conditions facilitate this high-resolution tracking. First, the unattenuated $100 \mathrm{~W}$ mercury-arc lamp is used for illumination. Mercury-arc has a strong emission peak at $546 \mathrm{~nm}$, which enables monitoring of DL549- and DL550-actin SiMS with a high signal-to-noise ratio. Typically, we acquire images at $100 \mathrm{~ms}$ intervals (fast tracking imaging, Fig. 3A) for the short duration of $10 \mathrm{~s}$. Special care must be taken to minimize the photodamage by restricting the illuminated area and duration of the fast tracking imaging. Second, subpixel localization of the SiMS centroid is determined by using the two-dimensional Gaussian fit model of Speckle TrackerJ. In practice, we first track a speckle by using the Constant-Velocity-NCC model of Speckle TrackerJ. Next we use the Adjustment model 5-10 times to refine the position of the speckle until the speckle mark stays in the same position. To see whether the position of speckle mark becomes stable, "Profiler" in the control panel helps. 
"Profiler" shows the intensity of a speckle for every frame that exists, and lines in the "Profiler" graph become stable as the speckle mark stays in the same position. The Adjustment model sometimes misidentifies speckles if other bright speckles or vesicles exist near the speckle in interest. Therefore, the position of speckle marks must be checked by eye and corrected manually when required. We then use the Gaussian-Fit model. The algorithm fits a Gaussian over a $2 \mathrm{~d}$ square (default $11 \times 11 \mathrm{px}^{2}$ ). It searches a size 0.75 pixels (default value) in $\mathrm{x}$ and $\mathrm{y}$ direction from the current speckle mark. In Speckle TrackerJ, iterating the algorithm several times refines the actual center of Gaussian of the speckle mark within 0.75 pixels. We typically apply the Gaussian-Fit model 3-4 times. To check how well the Gaussian-Fit model worked after using the model, you can select the menu "macros" and choose "Measure Speckle Tracks". It provides a table with information for the selected speckle in each frame. In the table, the value in the weight- 0 column is the standard deviation of the Gaussian. If the values in the weight- 1 and weight- 2 columns are zero at certain frames, the fit failed and the data are eliminated from the time series. Under the above conditions for SiMS imaging and tracking the speckle's centroid, the centroid of DL-actin SiMS can be resolved with a localization error of $\pm 8-8.5 \mathrm{~nm}$ (Yamashiro et al., 2014). This enables to reveal the velocity of actin filaments that move along the retrograde flow 100-150 nm that is within the diffraction limit of light microscope (Fig. 3B). Our results show that actin filaments undergo retrograde movement with a single flow speed regardless of their lifetimes in lamellipodia of XTC cells spread on a PLL-coated surface (Yamashiro et al., 2014).

(ii) Simultaneous analysis of actin dynamics with diverse timescales

DL-actin extends time window of the SiMS analysis, because photobleaching of DL549-actin is slow. Continuous exposure of the whole cell area to $1.5 \%$ of the mercury excitation for $600 \mathrm{sec}$ reduces DL549-actin fluorescence only to $76 \%$ of the original value (Fig. 2).

DL-actin enables simultaneous lifetime measurement of actin populations with markedly distinct disassembly kinetics. We compared the lifetime of DL549-actin speckles associating with actin stress fibers to that of speckles outside of stress fibers (Fig. 3C and D, Yamashiro et al., 2014). Stress fibers were visualized by Lifeact-EGFP (Fig. 3C). We carried out regression analysis to follow the surviving fraction of preexisting actin speckles (Watanabe, 2012). Double-exponential fits of the data suggest that speckles in both the stress fiber-associating and non-stress fiber filaments comprised two populations with distinct disassembly rates. Stress fiber-associated filaments disassembled at $t_{1 / 2}$ of $18.5 \mathrm{~s}(15 \%)$ and $t_{1 / 2}$ of $311.1 \mathrm{~s}(85 \%)$ (Fig. 3D, upper graph). Non-stress fiber filaments disassembled at $t_{1 / 2}$ of $32.3 \mathrm{~s}(73 \%)$ and $t_{1 / 2}$ of $233.5 \mathrm{~s}(27 \%)$ (Fig. 3D, lower graph). Thus, the majority of actin stress fibers disassemble with a half-life of 5 min, whereas 
a large fraction of non-stress fiber actin filaments disassemble within one minute.

The slow photobleaching of DL-actin would enable measurement of actin dynamics on diverse time scales, for instance, the formation and remodeling of thin filaments in myofibrils (Littlefield et al., 2001). DL-actin speckles are detectable for several days after electroporation, which is also advantageous to analyze the dynamics of actin structures during cell differentiation.

\section{Perspectives}

The SiMS microscopy introduced here is very easy to use live-cell single-molecule imaging. Furthermore, the new method achieves measurements of actin dynamics with a very high spatiotemporal resolution by using Speckle TrackerJ, which is a freely available open-source ImageJ plugin (http://athena.physics.lehigh.edu/speckletrackerj/index.chy).

Although DL-labeled probes provide substantially improved brightness and photostability, special care must still be taken to avoid photodamage to the cell in the SiMS microscopy. Intervals and duration of image acquisition as well as intensity and area of illumination must be determined empirically depending individual molecules of interest (Watanabe, 2012). The new SiMS microscopy will be applicable for monitoring single-molecules three-dimensionally, but tracking and analyzing molecular behavior while reducing photodamage during SiMS imaging in multiple focal planes might become hurdles to carry out the three-dimensional SiMS microscopy.

With the methodological advantage, the new SiMS microscopy will help researchers extend the application of single-molecule analysis. For instance, actin remodeling is the major output of signal transduction pathways activated by extracellular stimuli. Rho family GTPases (Heasman and Ridley, 2008), G-protein-coupled receptors (Cotton and Claing, 2009), the semaphorin receptors Plexins (Hung and Terman, 2011) and numerous other molecules play roles in regulating actin organization. Although the molecular mechanisms that regulate actin reorganization have well been characterized, spatiotemporal regulation of signaling from extracellular stimuli to actin still remains elusive. Thus direct viewing of actin dynamics provides clues to solve actin reorganization controlled by signaling molecules unambiguously.

The principles of the new SiMS method are applicable to many other proteins for monitoring at single-molecule level in live cells. If researchers develop a new probe for the present SiMS microscopy, we recommend confirming that labeling a protein of interest with a fluorescent dye does not disturb activities of the protein. In vitro labeling of the protein provides opportunities to characterize the properties of the labeled protein biochemically, which is advantage over the use of genetically-labeled proteins. With methodological advances introduced here, development of 
new probes may greatly extend SiMS imaging for various molecular dynamics.

\section{Acknowledgements}

This work was supported by the Human Frontiers Science Program Grant No. RGP0061/2009-C (N.W. and D.V.); NEXT program grant No. LS013 from the Cabinet Office, Government of Japan (N.W.); a grant from Takeda Science Foundation (N.W.) and a Grant-in-Aid for Scientific Research on Innovative Areas (S.Y.). 


\section{References}

Ananthanarayanan, V., Schattat, M., Vogel, S.K., Krull, A., Pavin, N., and Tolic-Norrelykke, I.M. (2013). Dynein motion switches from diffusive to directed upon cortical anchoring. Cell 153, 1526-1536.

Bovellan, M., Romeo, Y., Biro, M., Boden, A., Chugh, P., Yonis, A., Vaghela, M., Fritzsche, M., Moulding, D., Thorogate, R., Jegou, A., Thrasher, A.J., Romet-Lemonne, G., Roux, P.P., Paluch, E.K., and Charras, G. (2014). Cellular control of cortical actin nucleation. Curr Biol 24, 1628-1635.

Bubb, M. R., and Korn, E. D. (1995). Kinetic model for the inhibition of actin polymerization by actobindin. Biochemistry 34, 3921-3926.

Cai, D., McEwen, D.P., Martens, J.R., Meyhofer, E., and Verhey, K.J. (2009). Single molecule imaging reveals differences in microtubule track selection between kinesin motors. PLoS Biol 7, e1000216.

Chen, Q., Nag, S., and Pollard, T. D. (2012). Formins filter modified actin subunits during processive elongation. $J$ Struct Biol 177, 32-39.

Cotton, M., and Claing, A. (2009). G protein-coupled receptors stimulation and the control of cell migration. Cell Signal 21, 1045-1053.

Douglass, A.D., and Vale, R.D. (2005). Single-molecule microscopy reveals plasma membrane microdomains created by protein-protein networks that exclude or trap signaling molecules in $\mathrm{T}$ cells. Cell 121, 937-950.

Gebhardt, J.C., Suter, D.M., Roy, R., Zhao, Z.W., Chapman, A.R., Basu, S., Maniatis, T., and Xie, X.S. (2013). Single-molecule imaging of transcription factor binding to DNA in live mammalian cells. Nat Methods 10, 421-426.

Heasman, S. J., and Ridley, A. J. (2008). Mammalian Rho GTPases: new insights into their functions from in vivo studies. Nat Rev Mol Cell Biol 9, 690-701.

Houk, T. W., Jr., and Ue, K. (1974). The measurement of actin concentration in solution: a comparison of methods. Anal Biochem 62, 66-74.

Hung, R. J., and Terman, J. R. (2011). Extracellular inhibitors, repellents, and semaphorin/plexin/MICAL-mediated actin filament disassembly. Cytoskeleton (Hoboken) 68, 415-433.

Kan-o, M., Takeya, R., Taniguchi, K., Tanoue, Y., Tominaga, R., and Sumimoto, H. (2012). Expression and subcellular localization of mammalian formin Fhod3 in the embryonic and adult heart. PLoS One 7, e34765.

Kellogg, D. R., Mitchison, T. J., and Alberts, B. M. (1988). Behaviour of microtubules and actin filaments in living Drosophila embryos. Development 103, 675-686.

Leduc, C., Si, S., Gautier, J., Soto-Ribeiro, M., Wehrie-Haller, B., Gautreau, A., Giannone, G., Cognet, L., and Lounis, B. (2013). Nano Lett 13, 1489-1494. 
Littlefield, R., Almenar-Queralt, A., and Fowler, V. M. (2001). Actin dynamics at pointed ends regulates thin filament length in striated muscle. Nat Cell Biol 3, 544-551.

Millius, A., Watanabe, N., and Weiner, O. D. (2012). Diffusion, capture and recycling of SCAR/WAVE and Arp2/3 complexes observed in cells by single-molecule imaging. J Cell Sci 125, 1165-1176.

Millonig, R., Salvo, H., and Aebi, U. (1988). Probing actin polymerization by intermolecular cross-linking. J Cell Biol 106, 785-796.

Mizuno, H., Higashida, C., Yuan, Y., Ishizaki, T., Narumiya, S., and Watanabe, N. (2011). Rotational movement of the formin mDial along the double helical strand of an actin filament. Science 331, 80-83.

Ponti, A., Machacek, M., Gupton, S. L., Waterman-Storer, C. M., and Danuser, G. (2004). Two distinct actin networks drive the protrusion of migrating cells. Science 305, 1782-1786.

Schonichen, A., and Geyer, M. (2010). Fifteen formins for an actin filament: a molecular view on the regulation of human formins. Biochim Biophys Acta 1803, 152-163.

Severson, A. F., Baillie, D. L., and Bowerman, B. (2002). A Formin Homology protein and a profilin are required for cytokinesis and Arp2/3-independent assembly of cortical microfilaments in C. elegans. Curr Biol 12, 2066-2075.

Shinohara, R., Thumkeo, D., Kamijo, H., Kaneko, N., Sawamoto, K., Watanabe, K., Takebayashi, H., Kiyonari, H., Ishizaki, T., Furuyashiki, T., and Narumiya, S. (2012). A role for mDia, a Rho-regulated actin nucleator, in tangential migration of interneuron precursors. Nat Neurosci 15, 373-380, S371-372.

Smith, M. B., Karatekin, E., Gohlke, A., Mizuno, H., Watanabe, N., and Vavylonis, D. (2011). Interactive, computer-assisted tracking of speckle trajectories in fluorescence microscopy: application to actin polymerization and membrane fusion. Biophys $J$ 101, 1794-1804.

Spudich, J. A., and Watt, S. (1971). The regulation of rabbit skeletal muscle contraction. I. Biochemical studies of the interaction of the tropomyosin-troponin complex with actin and the proteolytic fragments of myosin. J Biol Chem 246, 4866-4871.

Vallotton, P., and Small, J. V. (2009). Shifting views on the leading role of the lamellipodium in cell migration: speckle tracking revisited. J Cell Sci 122, 1955-1958.

Watanabe, N. (2012). Fluorescence single-molecule imaging of actin turnover and regulatory mechanisms. Methods Enzymol 505, 219-232.

Watanabe, N., and Mitchison, T. J. (2002). Single-molecule speckle analysis of actin filament turnover in lamellipodia. Science 295, 1083-1086.

Watanabe, S., Ando, Y., Yasuda, S., Hosoya, H., Watanabe, N., Ishizaki, T., and Narumiya, S. (2008). mDia2 induces the actin scaffold for the contractile ring and stabilizes its position during cytokinesis in NIH 3 T3 cells. Mol Biol Cell 19, 2328-2338. 
Yamashiro, S., Mizuno, H., Smith, M. B., Ryan, G. L., Kiuchi, T., Vavylonis, D., and Watanabe, N. (2014). New single-molecule speckle microscopy reveals modification of the retrograde actin flow by focal adhesions at nanometer scales. Mol Biol Cell 25, 1010-1024.

Yamashiro, S. and Watanabe, N. A new link between the retrograde actin flow and focal adhesions. $J$ Biochem, in press. 


\section{Figure legends}

Figure 1 (A) DL549-actin coassembles to actin structures visualized by Lifeact-EGFP. An arrow in the DL549-actin image indicates a fluorescent organelle-like structure, which is apparently an artifact of elctroporation. Bar, $10 \mu \mathrm{m}$. (B) Images of DL549-actin speckles in lamellipodia of XTC cells. Time-lapse images paneled at $10 \mathrm{~s}$ intervals in the area (square) are shown on the right. Bar, $10 \mu \mathrm{m}$. DL549-actin speckles moving with the retrograde flow are indicated by colored circles. Modified from Yamashiro et al. 2014.

Figure 2 Photostability of DL549-actin in cells. XTC cells loaded with DL549-actin by electroporation were continuously exposed to excitation light using the $100 \mathrm{~W}$ mercury illumination system and acquired without interval. The excitation light used was full, attenuated to $25 \%$ or attenuated to $1.5 \%$. Black lines show the single-exponential curve fitted to the data. Relative intensity ( $y$-axis) expressed percentages derived from the average fluorescence intensity of the peripheral region of the XTC cell in each frame divided by the value at $y$-intercept of the fitted curve. Modified from Yamashiro et al. 2014.

Figure 3 Examples of the SiMS analysis with DL549-actin. (A) and (B), Nanometer-scale displacement analysis in cells. (A) Images of EGFP-Lifeact (left) or DL549-actin speckle acquired with a $100 \mathrm{~ms}$ exposure time and a full $100 \mathrm{~W}$ mercury excitation (right). Bar, $5 \mu \mathrm{m}$. (B) Displacement plot of the central position of a short-lived DL549-actin speckle in lamellipodia in the series of images acquired as (A). The velocity was calculated from a linear fit. (C) and (D), Simultaneous regression analyses of rapid and stable actin dynamics within and outside of stress fibers. (C) Merged image of DL-549 actin speckles (red) and stress fibers visualized by EGFP-Lifeact (green). White arrows indicate speckles in stress fibers. Blue arrows indicate speckles that were out of stress fibers. Bar, $5 \mu \mathrm{m}$. (D) Speckle regression analysis of speckles in stress fibers $(n=171)$ or out of stress fibers $(n=112)$ in 3 cells. Lines: the two-phase exponential curve fitted to the data gives $18.5 \mathrm{~s}(15 \%)$ and $311.1 \mathrm{~s}(85 \%)$ for the half-lives of speckles in stress fibers, whereas it gives $32.3 \mathrm{~s}(73 \%)$ and $233.5 \mathrm{~s}(27 \%)$ for speckles outside of stress fibers. Modified from Yamashiro et al. 2014. 


\section{Lifeact-EGFP}

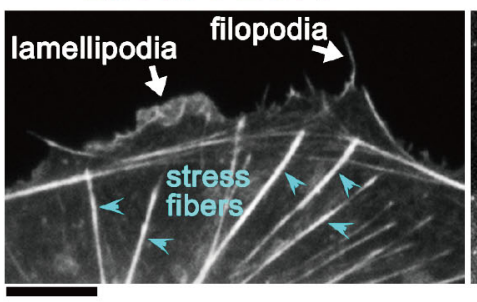

DL549-actin

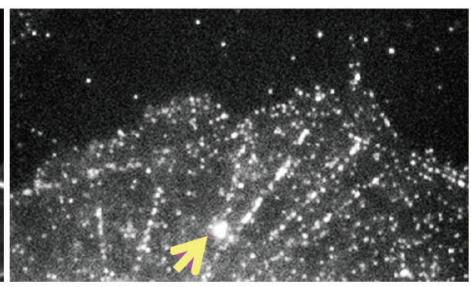

Merged

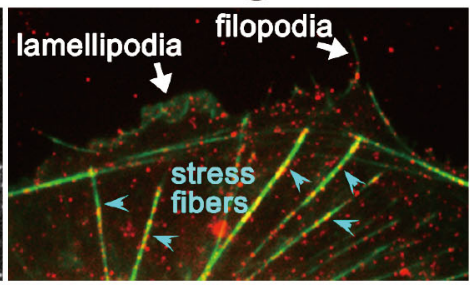

B
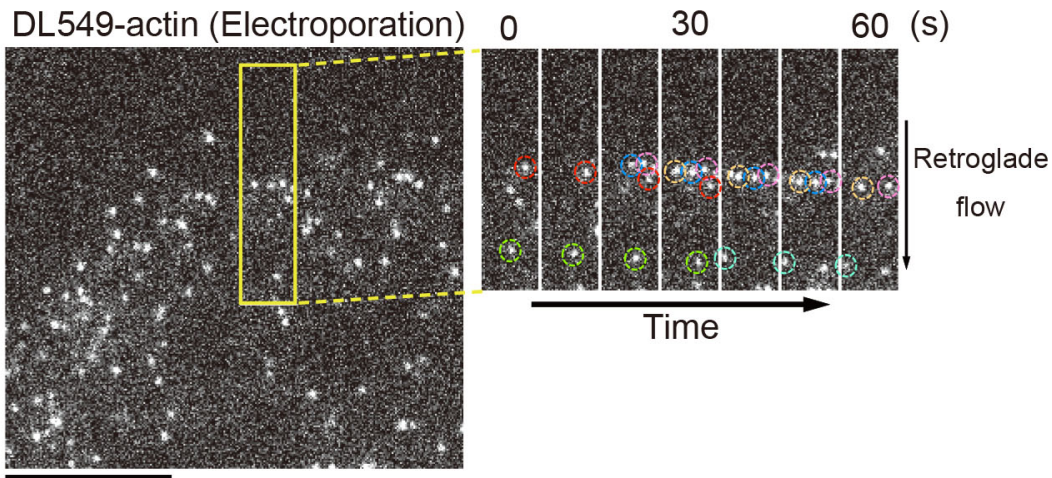


\section{F. 京都大学。}

KYOTO UNIVERSITY https://repository.kulib.kyoto-u.ac.jp

\section{Photostability of DL549-actin in vivo}

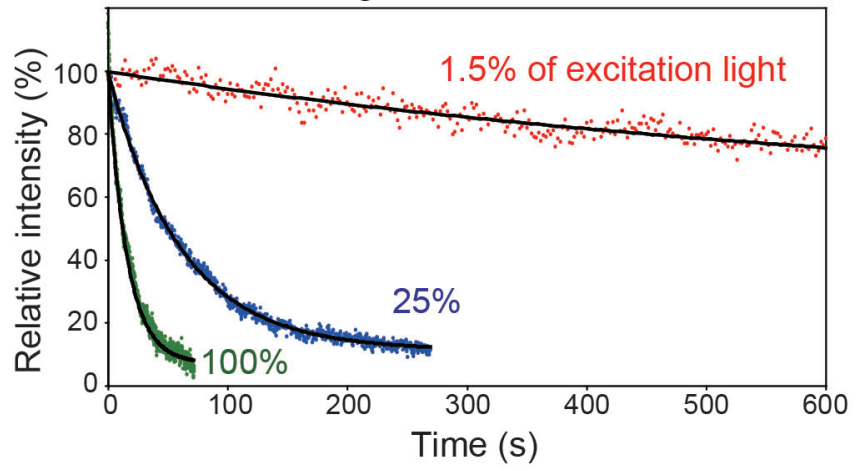


A

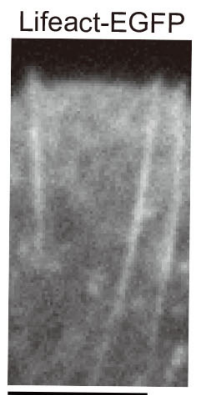

$100 \mathrm{~ms}$ exposure,

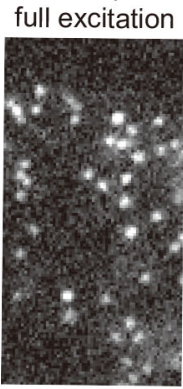

C Lifeact-EGFP

DL549-actin

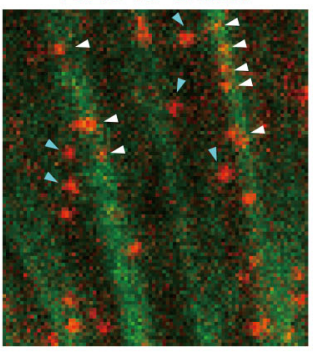

B Speckle in lamellipodia

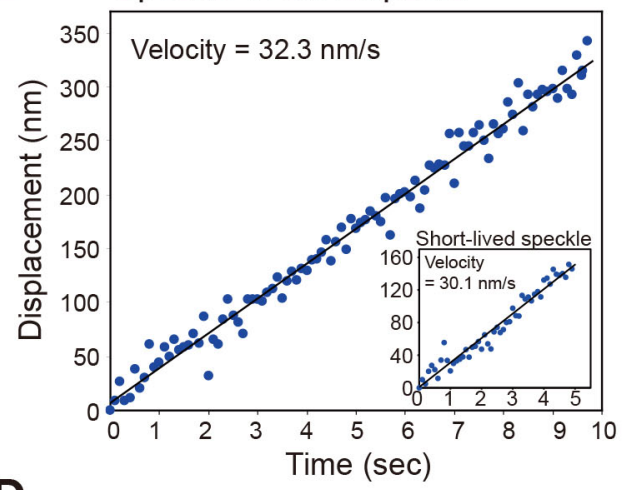

Regression analysis
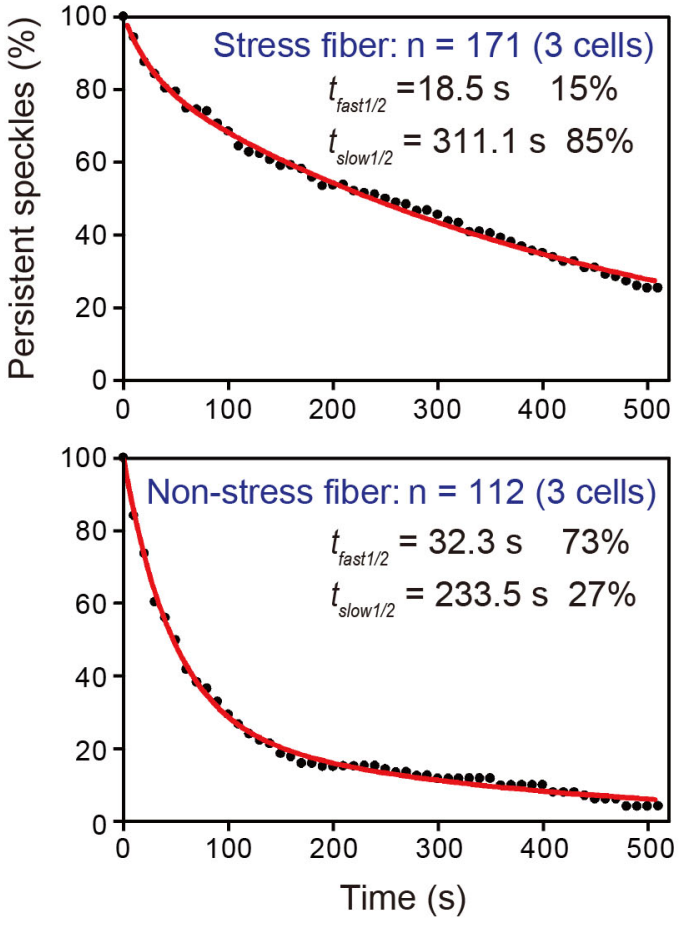